\title{
EFFECT OF STORAGE PERIODS, CULTIVARS, ENVIRONMENTS AND PACKAGE MATERIALS ON GERMINATION, VIABILITY AND SEEDLING VIGOR OF WHEAT GRAINS
}

\author{
Omar, A. M.* ; F. A. Sorour * ; Soad A. El-Sayed ${ }^{* *}$ and \\ Nagwa. E. Shalaby. * \\ * Agronomy Dep., Faculty of Agriculture, Kafrelsheikh University. \\ ** Seed Technology Division, Field Crop Res. Institute, ARC, Egypt.
}

\begin{abstract}
An experiment was carried out at Sakha Agricultural Research Station during 2010 and 2011 seasons to study the effect of storage periods, cultivars, environments and package materials on germination, viability and seedling vigor of wheat grains.

Three cultivars of wheat (Gemmiza7, Sakha 93 and Giza 168) were used during this study. Grains were packaged in three package materials (Jute, Jute coated plastic, Metal cans) after that grains stored in three types of environments (Dust, Sand and Ash). The stored grains were tested after two different periods for germination, viability (Electrical conductivity and Acidity \%) and seedling vigor (radical length, plumule length and seedling dry weight). The obtained results recorded highly significant differences among the tested cultivars for all studied characters. Moreover, increasing storage period after harvest until 18 months decreased significantly all characters except electrical conductivity and acidity percentage which were increased with increasing storage period. Also, highly significant differences were observed among grains quality characters due to the different storage environments. Grains stored in ash had the worst characters as compared with other environments while grains stored in dust and sand gave the best characters. Moreover, grains stored in metal cans produced the best characters while, grains stored in jute bags gave the worst characters. It could be recommended that using metal cans to store wheat grains tell 18 months without using any chemicals is the best method for saving viability and seedling vigor.
\end{abstract}

\section{INTRODUCTION}

The purpose of seed storage is to preserve planting stocks from one season to the next. In some cases, the objective of seed storage is to maintain seed quality for the longest duration possible to be utilizing for nutritional consumption. This approach creates a greater diversity in seed inventory and provides a guarantee to seed supply in years when acceptable seed quality and production is low (Thamaga-Chitja et al., 2004). In addition, seed storage enables the maintenance of germplasm over time for improved plant breeding programs (Copeland and McDonald, 1995).

The major determinants of storage risk are moisture, temperature, type of storage bags and time of storage (Hong et al., 1994). In some parts of the world, especially in the tropics, conditioned storage is necessary in order to maintain high viability of some seed from harvest to planting (Harrington, 1970). 
In recent years, packaging seeds in moisture-barrier containers to prevent loss of viability and resistant or hermitically sealed containers for storage and marketing has explored. The purpose of such containers is to maintain seeds at safe storage moisture levels (Copeland and McDonald, 1995). Seeds in package that are not completely impervious to moisture may gain or lose moisture with time. Seeds in small packages gain or lose moisture faster than seeds in large packages. Therefore, small packages require better moisture-barrier materials than do large ones for equal moisture protection (Bass, 1971).

Despite significant advances in food storage methods, many African and South African communities still rely on traditional storage methods for seed to be used as food and fodder (Olakojo and Akinlosotu,2004 and Thamaga-Chitja et al., 2004) because 1- Chemical insecticides have serious drawbacks such as genetic resistance, toxic residues, worker safety, increasing costs of application and decreasing seed viability. 2- Small-scale farmers may not have facilities to store their seed at $4-10^{\circ} \mathrm{C}$.

The aim of the present investigation was to study the effect of storage periods, varieties, environments and package materials on germination, viability and seedling vigor of wheat grains.

\section{MATERIALS AND METHODS}

This investigation was carried out in the laboratory of Seed Technology at Sakha Agricultural Research Station, Kafr El-Sheikh during the period from May, (2010) to November, (2011) to study the effect of two storage periods [6 months (A1) and 18 months (A2)], three wheat cultivars [Gemmeza 7(B1), Sakha 93(B2) and Giza 168(B3)], three storage environments [Dust (C1), Sand (C2) and Ash (C3)] and three package materials [Jute (D1), Jute coated plastic (D2) and Cans (D3)] on germination, viability and seedling vigor of wheat grains. Factorial design in completely randomized (CRD) was used (Gomez and Gomez, 1984).

Samples of wheat grains were obtained from the season of 2010 after harvesting (15 kg from each cultivar). The samples were sieved and cleaned from dust, husk or any inert materials then divided into three portions. The first portion was kept without storage. The second portion was packaged in [Jute (D1), Jute coated plastic (D2) and metal cans (D3)]. Each package was filled with $500 \mathrm{~g}$ of seeds and stored in three different environments [Dust (C1), Sand (C2) and Ash (C3)]. The third portion was the same of the second portion. Random grain samples were taken from each package at two different periods of storage [after 6 months of storage (A1) and after 18 months of storage (A2)].

Data were recorded on standard germination:- Test was carried out under optimum conditions according to international rules testing (ISTA, 2006).

Acidity percentage:- Grain samples were taken at random from each plot and grounded to fine powder to pass through $2 \mathrm{~mm}$ mesh to determine acidity percentage according to (AOAC, 1999). 
At the beginning of storage germination $\%$, viability and seedling vigor of wheat grains were measured on an original weight basis as follows in table 1

Table 1: Germination, viability and seedling vigor of wheat grains as measured before storing.

\begin{tabular}{|l|c|c|c|}
\hline \multicolumn{1}{|c|}{ Characters studied } & \multicolumn{3}{c|}{ Wheat } \\
\cline { 2 - 4 } & Gemiza7 & Sakha.93 & Giza168 \\
\hline Germination $(\%)$ & 100 & 100 & 100 \\
\hline Conductivity $(\mu$-mhos/g) & 13 & 13.9 & 13.4 \\
\hline Acidity $(\%)$ & 0.20 & 0.22 & 0.21 \\
\hline Radical length $(\mathrm{cm})$ & 14 & 14.5 & 14.2 \\
\hline Plumule length $(\mathrm{cm})$ & 15 & 15.7 & 15.3 \\
\hline Seedling dry weight $(\mathrm{mg})$ & 24 & 24 & 24 \\
\hline
\end{tabular}

\section{RESULTS AND DISCUSSION}

Increasing storage period significantly decreased germination percentage, radical length, plumule length and seedling dry weight. The decline in these traits was associated with an increase in electrical conductivity values and acidity percentage (Table 2). Similar results were reported by El-Aidy (1988), El-Borai et al. (1993), Soad (1997), El-Aidy et al. (2001) and El-Sayed et al. (2004a).

A highly significant difference among studied cultivars for all traits were obtained. Sakha 93 was significantly lower in germination percentage, radical length and higher in electrical conductivity and acidity percentage(Table 2).

For storage environments highly significant differences were obtained in all tested characters. Grains stored in ash gave the lowest germination percentage, radical length, plumule length and seedling dry weight ,while it gave the highest values in electrical conductivity and acidity percentage. On the other hand grains stored in dust and sand gave higher values in germination percentage, radical length, plumule length and lower values in electrical conductivity and acidity percentage ( Table 2).

Data cleared that grains stored in metal cans significantly superior to the other two package materials. Grains stored in cans gave higher values in germination percentage, radical length, plumule length, seedling dry weight and lower values in electrical conductivity and acidity percentage. These results are in harmony with those reported by Soad (1997). 
Omar, A. M. et al.

Table 2: Germination, viability and seedling vigor of wheat grains as affected by storage periods, cultivars, environments, package materials and their interactions.

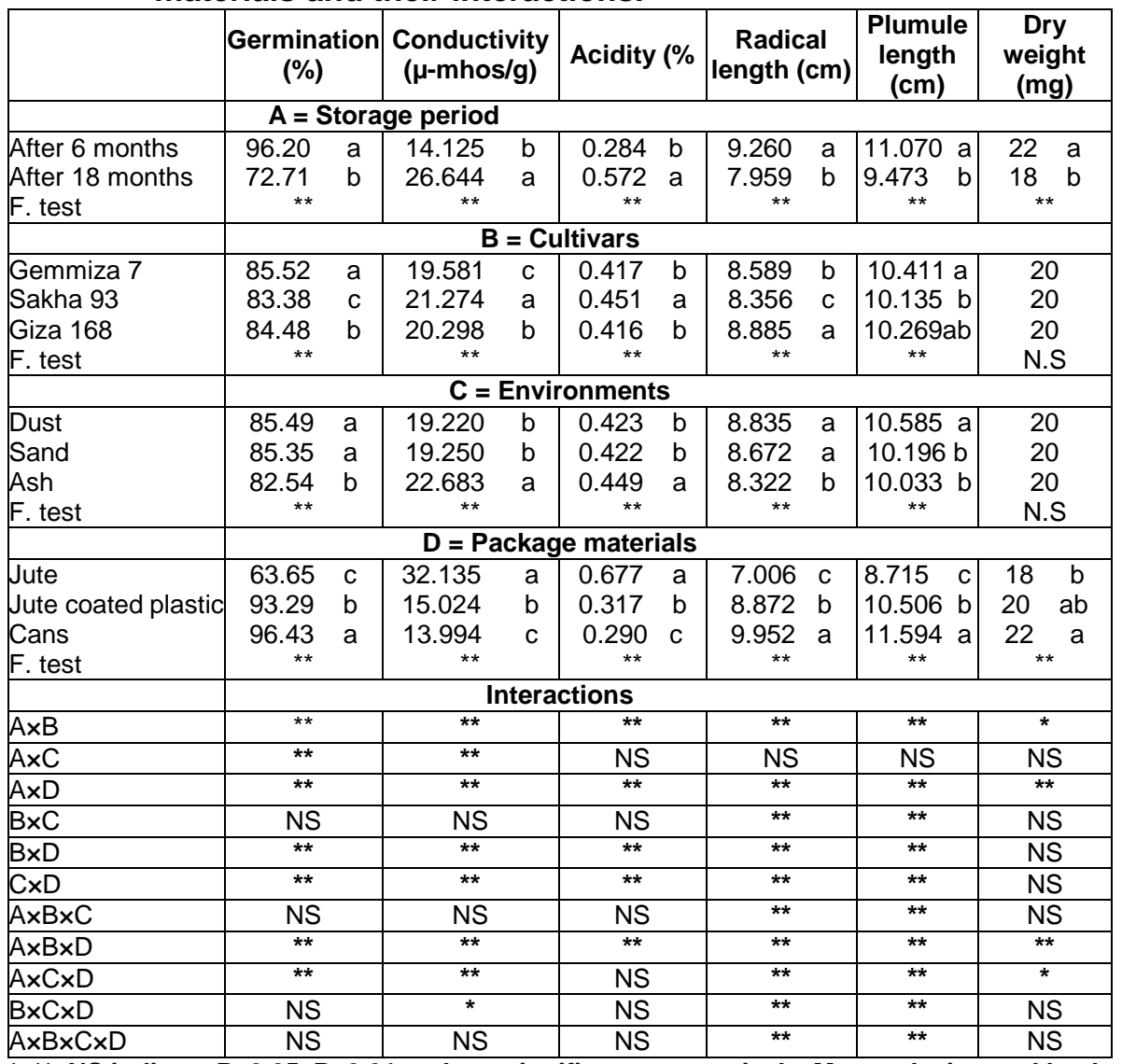

${ }^{\star},{ }^{\star *}$, NS indicate $\mathbf{P}<0.05, P<0.01$ and not significant, respectively. Means designated by the same letter within columns are not significantly different according to Duncan's multiple range test.

The interaction between storage periods and cultivars indicated highly significant differences in all studied characters (Table 3). Data clear that increasing storage period significantly decreased germination percentage and seedling vigor with all cultivars. On the other hand increasing storage period significantly increased electrical conductivity values and acidity percentage. These results are in harmony with those reported by El-Sayed and Abd El-Aziz (2005) and Govender et al. (2007). 
Table 3: Germination, viability and seedling vigor of wheat grains as affected by the interaction between storage periods and cultivars.

\begin{tabular}{|c|c|c|c|c|c|c|c|c|c|c|}
\hline \multirow[t]{2}{*}{ Periods } & \multirow{2}{*}{\begin{tabular}{|l|} 
Cultivars \\
B1 \\
\end{tabular}} & \multirow{2}{*}{\begin{tabular}{|l} 
Germ. \\
96.7 a
\end{tabular}} & \multirow{2}{*}{\begin{tabular}{|c|} 
E.C \\
$13.57 \mathrm{e}$ \\
\end{tabular}} & \multirow{2}{*}{$\begin{array}{l}\text { Acidity } \\
0.28 \text { cd }\end{array}$} & \multicolumn{2}{|c|}{$\begin{array}{c}\text { Radical } \\
\text { Length }(\mathrm{cm})\end{array}$} & \multicolumn{2}{|c|}{$\begin{array}{c}\text { Plumule } \\
\text { Length }(\mathrm{cm})\end{array}$} & \multicolumn{2}{|c|}{$\begin{array}{c}\text { Seedling } \\
\text { dry weight }(\mathrm{mg})\end{array}$} \\
\hline & & & & & 9.33 & $a$ & 11.37 & $a$ & 23 & $a$ \\
\hline \multirow[t]{2}{*}{ A1 } & B2 & $95.7 \mathrm{a}$ & $14.59 \mathrm{~d}$ & 0.3 & 9.37 & $a$ & 11.05 & $\mathrm{~b}$ & 23.4 & $\mathrm{a}$ \\
\hline & B3 & $96.2 \mathrm{a}$ & $14.20 \mathrm{~d}$ & $0.272 \mathrm{~d}$ & 9.09 & $a$ & 10.79 & $\mathrm{C}$ & 23.2 & $a$ \\
\hline \multirow{3}{*}{ A2 } & B1 & $74.3 \mathrm{~b}$ & $25.59 \mathrm{c}$ & $0.554 \mathrm{~b}$ & 7.85 & $C$ & 9.46 & $d$ & 18 & $\mathrm{~b}$ \\
\hline & B2 & $65.2 \mathrm{~d}$ & $27.95 \mathrm{a}$ & $0.601 \mathrm{a}$ & 7.34 & $d$ & 9.49 & $d$ & 17.6 & $b$ \\
\hline & B3 & $72.7 \mathrm{c}$ & $26.39 \mathrm{~b}$ & $0.561 \mathrm{~b}$ & 8.68 & $b$ & 9.47 & $d$ & 18.6 & $b$ \\
\hline \multicolumn{2}{|l|}{ Sig. } & ** & ** & $\star *$ & ** & & $\star \star$ & & * & \\
\hline
\end{tabular}

${ }^{*},{ }^{* \star}$, NS indicate $\mathrm{P}<0.05, \mathrm{P}<0.01$ and not significant, respectively. Means designated by the same letter within columns are not significantly different according to Duncan's multiple range test.

The interaction between storage periods and environments indicated significantly affected germination percentage and electrical conductivity values (Table 4). Results clear that grains stored in ash gave the lowest germination percentage and the highest electrical conductivity value. In contrast grains stored in dust and sand gave the highest germination percentage and the lowest electrical conductivity value during the two storage periods. These results are in agreement with those recorded by Soares et al. (1993) and Tippes (1995).

Table 4: Germination and electrical conductivity of wheat grains as affected by the interaction between storage periods and environments.

\begin{tabular}{|c|c|c|c|c|}
\hline Periods & Environments & Germ. & E.C & \\
\hline \multirow{3}{*}{ A1 } & C1 & $96.6 \quad \mathrm{a}$ & 14.08 & $\mathrm{C}$ \\
\hline & $\mathrm{C} 2$ & $96.5 \mathrm{a}$ & 14.07 & C \\
\hline & C3 & $95.5 \mathrm{a}$ & 14.22 & $\mathrm{C}$ \\
\hline \multirow{3}{*}{ A2 } & C1 & $74.4 \quad b$ & 24.36 & $\mathrm{~b}$ \\
\hline & $\mathrm{C2}$ & $74.2 \quad b$ & 24.43 & $b$ \\
\hline & C3 & 62 & 31.15 & $\mathrm{a}$ \\
\hline \multicolumn{2}{|l|}{ Sig. } & ** & \multicolumn{2}{|l|}{ ** } \\
\hline
\end{tabular}

${ }^{*},{ }^{* *}$, NS indicate $\mathbf{P}<0.05, P<0.01$ and not significant, respectively. Means designated by the same letter within columns are not significantly different according to Duncan's multiple range test.

Highly significant interaction was obtained between storage periods and package materials in all studied characters (Table 5). Results showed that grains stored in metal cans remained higher values in germination percentage, seedling vigor and lower values in electrical conductivity and acidity. On the other hand, grains stored in jute bags gave lower values in germination percentage, seedling vigor and higher values in electrical conductivity and acidity during the two storage periods. Similar results were obtained by El-Aidy et al. (2001) and El-Sayed (2004b). 
Omar, A. M. et al.

Table 5: Germination, viability and seedling vigor of wheat grains as affected by the interaction between storage periods and package materials.

\begin{tabular}{|c|c|c|c|c|c|c|c|}
\hline Periods & $\begin{array}{l}\text { Package } \\
\text { materials }\end{array}$ & Germ. & E.C & Acidity & $\begin{array}{c}\text { Radical } \\
\text { length }(\mathrm{cm})\end{array}$ & $\begin{array}{c}\text { Plumule } \\
\text { length }(\mathrm{cm})\end{array}$ & \begin{tabular}{|c|} 
Seedling \\
dry weight(mg)
\end{tabular} \\
\hline \multirow{3}{*}{ A1 } & D1 & $93.3 \mathrm{~d}$ & $14.42 \quad \mathrm{c}$ & $0.342 \mathrm{c}$ & $8.59 \mathrm{~d}$ & $9.99 \mathrm{C}$ & $21.8 \mathrm{ab}$ \\
\hline & D2 & $97.2 \quad b$ & $14.09 \mathrm{~cd}$ & $0.269 \mathrm{~d}$ & 8.92 & $11.26 \mathrm{~b}$ & 23.8 a \\
\hline & D3 & $98.2 \mathrm{a}$ & $13.86 \mathrm{~d}$ & $0.24 \mathrm{e}$ & $10.27 \quad \mathrm{a}$ & $11.96 \quad \mathrm{a}$ & 24 \\
\hline \multirow{3}{*}{$\mathrm{A} 2$} & D1 & 34 & 49.85 a & $1.011 \mathrm{a}$ & 5.42 & 7.44 & $14.8 \mathrm{C}$ \\
\hline & D2 & 89.4 e & $15.96 \mathrm{~b}$ & $0.366 \mathrm{~b}$ & $8.83 \mathrm{~cd}$ & 9.75 & $17.2 \mathrm{bc}$ \\
\hline & D3 & $94.7 \quad c$ & $14.13 \mathrm{~cd}$ & $0.34 \quad c$ & $9.63 \quad b$ & $11.23 \mathrm{~b}$ & 22 \\
\hline Sig. & & ** & $\star *$ & ** & ** & $\star *$ & ** \\
\hline
\end{tabular}

${ }^{*},{ }^{*}$, NS indicate $\mathrm{P}<0.05, \mathrm{P}<0.01$ and not significant, respectively. Means designated by the same letter within columns are not significantly different according to Duncan's multiple range test.

Data clear that Gemmiza 7 stored in dust gave the highest value in radical and plumule lengths while, Giza 168 had the lowest value of radical and plumule lengths when stored in ash environment (Table 6). Similar results were reported by El-Aidy et al. (2001) and El-Sayed et al. (2004a).

Table 6: Radical and plumule lengths of wheat grains as affected by the interaction between cultivars and storage environments.

\begin{tabular}{|c|c|c|c|}
\hline Cultivars & Environments & $\begin{array}{c}\text { Radical } \\
\text { Length }(\mathbf{c m})\end{array}$ & $\begin{array}{l}\text { Plumule } \\
\text { Length }(\mathrm{cm})\end{array}$ \\
\hline \multirow{3}{*}{ B1 } & C1 & $8.65 \mathrm{bc}$ & $10.767 \mathrm{a}$ \\
\hline & $\mathrm{C} 2$ & $8.461 \mathrm{~cd}$ & $10.178 \mathrm{bcd}$ \\
\hline & C3 & $8.656 \mathrm{bc}$ & $10.289 \mathrm{bc}$ \\
\hline \multirow{3}{*}{ B2 } & C1 & $8.583 \mathrm{bc}$ & $10.594 \mathrm{ab}$ \\
\hline & $\mathrm{C} 2$ & $8.472 \mathrm{~cd}$ & $10.394 \mathrm{abc}$ \\
\hline & C3 & $8.011 \mathrm{~d}$ & $9.817 \mathrm{~d}$ \\
\hline \multirow{3}{*}{ B3 } & C1 & $9.272 \mathrm{a}$ & $10.394 \mathrm{abc}$ \\
\hline & $\mathrm{C} 2$ & $9.083 \mathrm{ab}$ & $10.017 \mathrm{~cd}$ \\
\hline & C3 & $8.3 \mathrm{~cd}$ & $9.994 \mathrm{~cd}$ \\
\hline Sig & & 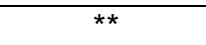 & - \\
\hline
\end{tabular}

${ }^{\star},{ }^{\star *}, \mathrm{NS}$ indicate $\mathrm{P}<0.05, \mathrm{P}<0.01$ and not significant, respectively. Means designated by the same letter within columns are not significantly different according to Duncan's multiple range test.

Highly significant differences were found in all traits except seedling dry weight to the interaction between cultivars and package materials (Table7). Results indicated that grains stored in metal cans gave higher values in germination percentage, radical length, plumule length and lower values in electrical conductivity value and acidity percentage with all cultivars. In contrast grains stored in jute bags gave lower values in germination percentage, radical length, plumule length and higher values in electrical conductivity and acidity percentage with all cultivars. These results are in harmony with those recorded by El-Aidy (1988), El-Aidy et al. (2001) and ElSayed et al.(2004b). 
Table 7: Germination, viability, radical and plumule lengths of wheat grains as affected by the interaction between cultivars and package materials.

\begin{tabular}{|c|c|c|c|c|c|c|}
\hline cultivars & $\begin{array}{l}\text { Package } \\
\text { materials }\end{array}$ & Germ. & E.C & Acidity & $\begin{array}{c}\text { Radical } \\
\text { Length }(\mathrm{cm})\end{array}$ & $\begin{array}{c}\text { Plumule } \\
\text { Length }(\mathrm{cm})\end{array}$ \\
\hline \multirow{3}{*}{ B1 } & D1 & $65.8 \mathrm{c}$ & $30.43 \mathrm{c}$ & $0.667 \mathrm{~b}$ & $6.83 \mathrm{~d}$ & $8.5 \quad f$ \\
\hline & $\mathrm{D} 2$ & 94 & 14.64 ef & $0.308 \mathrm{~cd}$ & $8.89 \quad b$ & $10.74 \mathrm{c}$ \\
\hline & D3 & $96.8 \quad \mathrm{a}$ & $13.67 \mathrm{~h}$ & $0.276 \mathrm{e}$ & $10.05 \mathrm{a}$ & $11.99 \mathrm{a}$ \\
\hline \multirow{3}{*}{ B2 } & D1 & 61 & $34.16 \mathrm{a}$ & $0.718 \mathrm{a}$ & $6.18 \mathrm{e}$ & $8.53 \mathrm{f}$ \\
\hline & $\mathrm{D} 2$ & $92.9 \mathrm{~b}$ & $15.37 \mathrm{~d}$ & $0.329 \mathrm{c}$ & $8.96 \mathrm{~b}$ & $10.76 \mathrm{c}$ \\
\hline & D3 & $96.2 \mathrm{a}$ & $14.3 \mathrm{fg}$ & $0.305 \mathrm{~cd}$ & $9.922 \mathrm{a}$ & $11.52 b$ \\
\hline \multirow{3}{*}{ B3 } & D1 & $64.2 \mathrm{~d}$ & $31.82 \mathrm{~b}$ & $0.645 \mathrm{~b}$ & $8.01 \mathrm{C}$ & $9.11 \mathrm{e}$ \\
\hline & D2 & $93 \quad b$ & $15.06 \mathrm{de}$ & $0.314 \mathrm{~cd}$ & $8.77 \quad b$ & $10.02 \mathrm{~d}$ \\
\hline & D3 & $96.3 \quad \mathrm{a}$ & $14.01 \mathrm{gh}$ & $0.289 \mathrm{de}$ & $9.883 \mathrm{a}$ & $11.28 \mathrm{~b}$ \\
\hline \multicolumn{2}{|l|}{ Sig. } & $\star \star *$ & ** & ** & $\star * *$ & ** \\
\hline
\end{tabular}

${ }^{*},{ }^{\star *}$, NS indicate $P<0.05, P<0.01$ and not significant, respectively. Means designated by the same letter within columns are not significantly different according to Duncan's multiple range test.

ighly significant differences were found in all traits except seedling dry weight due to the interaction between storage environments and package materials (Table8). Data showed that grains stored in jute bags and ash environment gave lower values in germination percentage, radical length, plumule length and higher values in electrical conductivity and acidity percentage, while grains stored in metal cans and dust or sand gave higher values in germination percentage, radical length, plumule length and lower values in electrical conductivity value and acidity percentage. Similar results were recorded by El-Sayed et al. (2004b).

Table 8: Germination, viability, radical and plumule lengths of wheat grains as affected by the interaction between storage environments and package materials.

\begin{tabular}{|c|c|c|c|c|c|c|}
\hline Environments & \begin{tabular}{|l|} 
Package \\
materials
\end{tabular} & Germ. & E.C & Acidity & $\begin{array}{c}\text { Radical } \\
\text { Length }(\mathrm{cm})\end{array}$ & $\begin{array}{c}\text { Plumule } \\
\text { Length(cm) }\end{array}$ \\
\hline \multirow{3}{*}{ C1 } & $\mathrm{D} 1$ & $65.8 \mathrm{~d}$ & $28.79 \mathrm{~b}$ & $0.668 \quad b$ & $7.61 \mathrm{~d}$ & $9.333 \mathrm{~d}$ \\
\hline & $\mathrm{D} 2$ & $93.9 \mathrm{~b}$ & $14.89 \mathrm{c}$ & $0.309 \mathrm{~cd}$ & $8.92 \mathrm{~b}$ & $10.81 \mathrm{~b}$ \\
\hline & D3 & 96.6 a & $13.98 \mathrm{~d}$ & $0.291 \mathrm{~d}$ & $9.97 \mathrm{a}$ & $11.62 \mathrm{a}$ \\
\hline \multirow{3}{*}{ C2 } & $\mathrm{D} 1$ & $65.9 \mathrm{~d}$ & $28.84 \mathrm{~b}$ & $0.664 \mathrm{~b}$ & $6.91 \mathrm{e}$ & 8.63 \\
\hline & $\mathrm{D} 2$ & $93.9 \mathrm{~b}$ & $14.91 \mathrm{c}$ & $0.312 \mathrm{~cd}$ & $9.19 \mathrm{~b}$ & $10.43 \mathrm{c}$ \\
\hline & D3 & $96.2 \mathrm{a}$ & 14 & $0.289 \mathrm{~d}$ & $9.92 \mathrm{a}$ & $11.53 \quad \mathrm{a}$ \\
\hline \multirow{3}{*}{ C3 } & $\mathrm{D} 1$ & $59.2 \mathrm{e}$ & $38.78 \mathrm{a}$ & $0.697 \mathrm{a}$ & $6.49 \mathrm{f}$ & $8.183 \mathrm{f}$ \\
\hline & $\mathrm{D} 2$ & 92 & $15.27 \mathrm{c}$ & $0.329 \mathrm{c}$ & $8.51 \mathrm{C}$ & $10.28 \mathrm{c}$ \\
\hline & D3 & $96.4 \mathrm{a}$ & 14 & $0.289 \mathrm{~d}$ & $9.97 \mathrm{a}$ & $11.64 \quad \mathrm{a}$ \\
\hline Sig. & & ** & ** & ** & ** & ** \\
\hline
\end{tabular}

${ }^{*},{ }^{* *}$, NS indicate $P<0.05, P<0.01$ and not significant, respectively. Means designated by the same letter within columns are not significantly different according to Duncan's multiple range test.

adical and plumule lengths significantly affected by the interaction among storage periods, cultivars and environments ( Table 9). Sakha 93 
Omar, A. M. et al.

stored in ash environment had the lowest radical and plumule length after 18 months while, grains stored in dust environment gave the highest radical length for Sakha 93 after 6 months but, Gemmiza 7 had the highest value for plumule length when grains stored in dust environment ( Table 9). Similar results were reported Soad (1997), El-Aidy et al.(2001) and El-Sayed et al. (2004b).

Table 9: Radical and plumule lengths of wheat grains as affected by the interaction between storage periods and cultivars.

\begin{tabular}{|c|c|c|c|c|}
\hline Periods & Cultivars & Environments & $\begin{array}{c}\text { Radical } \\
\text { Length(cm) }\end{array}$ & $\begin{array}{c}\text { Plumule } \\
\text { Length }(\mathrm{cm})\end{array}$ \\
\hline \multirow{9}{*}{ A1 } & B1 & C1 & $9.24 \mathrm{de}$ & $11.58 \quad \mathrm{a}$ \\
\hline & B1 & $\mathrm{C} 2$ & $9.47 \mathrm{bc}$ & $11.38 \mathrm{~b}$ \\
\hline & $\mathrm{B} 1$ & C3 & $9.27 \mathrm{~cd}$ & $11.14 \mathrm{~cd}$ \\
\hline & $\mathrm{B} 2$ & C1 & $10.03 \mathrm{a}$ & $11.26 \mathrm{bc}$ \\
\hline & B2 & $\mathrm{C} 2$ & $9.3 \mathrm{bcd}$ & $11.17 \mathrm{~cd}$ \\
\hline & $\mathrm{B} 2$ & C3 & $8.77 \mathrm{~g}$ & $10.72 \mathrm{e}$ \\
\hline & B3 & C1 & $9.5 \mathrm{~b}$ & $11.07 \mathrm{~d}$ \\
\hline & B3 & $\mathrm{C} 2$ & 9.17 def & $10.59 \mathrm{e}$ \\
\hline & B3 & C3 & $8.6 \mathrm{~g}$ & $10.73 \mathrm{e}$ \\
\hline \multirow{9}{*}{ A2 } & B1 & C1 & $8.06 \mathrm{~h}$ & $9.96 \mathrm{f}$ \\
\hline & B1 & C2 & $7.46 \quad \mathrm{i}$ & $8.98 \mathrm{j}$ \\
\hline & $\mathrm{B} 1$ & C3 & $8.04 \mathrm{~h}$ & $9.43 \mathrm{~h}$ \\
\hline & $\mathrm{B} 2$ & C1 & $7.13 \mathrm{j}$ & $9.93 \mathrm{f}$ \\
\hline & B2 & C2 & $7.64 \mathrm{i}$ & $9.62 \mathrm{~g}$ \\
\hline & B2 & C3 & $7.26 \mathrm{j}$ & 8.91 j \\
\hline & B3 & C1 & 9.04 ef & $9.72 \mathrm{~g}$ \\
\hline & B3 & C2 & 9 & $9.44 \mathrm{~h}$ \\
\hline & B3 & C3 & 8 & $9.26 \mathrm{i}$ \\
\hline Siq. & & & $* *$ & ** \\
\hline
\end{tabular}

${ }^{*},{ }^{\star *}$, NS indicate $\mathrm{P}<0.05, \mathrm{P}<0.01$ and not significant, respectively. Means designated by the same letter within columns are not significantly different according to Duncan's multiple range test.

The interaction among storage periods, cultivars and package materials indicated highly significant differences in all traits (Table 10). Data showed that grains stored in jute bags gave lower values in germination percentage, seedling vigor and higher values in electrical conductivity and acidity percentage with all cultivars especially sakha 93 while grains stored in metal cans gave higher values in germination percentage, seedling vigor and lower values in electrical conductivity and acidity percentage with all cultivars especially Gemmiza 7. These results are in harmony with those recorded by El-Aidy (1988), El-Aidy et al. (2001) and El-Sayed et al.(2004a). 
Table 10: Germination, viability and seedling vigor of wheat grains as affected by the interaction among storage periods, cultivars and package materials.

\begin{tabular}{|c|c|c|c|c|c|c|c|c|}
\hline Periods & Cultivars & $\begin{array}{l}\text { Package } \\
\text { materials }\end{array}$ & Germ. & E.C & Acidity & $\begin{array}{c}\text { Radical } \\
\text { Length }(\mathrm{cm})\end{array}$ & $\begin{array}{l}\text { Plumule } \\
\text { Length }(\mathrm{cm})\end{array}$ & $\begin{array}{c}\text { Seedling } \\
\text { dry weight }(\mathrm{mg})\end{array}$ \\
\hline \multirow{9}{*}{ A1 } & B1 & D1 & $93.9 \mathrm{bc}$ & $13.86 \mathrm{fgh}$ & $0.336 \mathrm{de}$ & 8.91 cde & $10.34 \mathrm{fg}$ & $22 \quad a b c$ \\
\hline & $\mathrm{B} 1$ & D2 & $97.7 \mathrm{a}$ & $13.54 \mathrm{gh}$ & $0.27 \mathrm{fg}$ & $9.07 \mathrm{bcd}$ & $11.51 \mathrm{bc}$ & $23.8 \mathrm{ab}$ \\
\hline & B1 & D3 & $98.5 \mathrm{a}$ & $13.32 \mathrm{~h}$ & $0.232 \mathrm{~g}$ & $10 \quad a$ & $12.24 \mathrm{a}$ & $23.4 \mathrm{ab}$ \\
\hline & B2 & D1 & $92.4 \mathrm{c}$ & $14.87 \mathrm{e}$ & $0.371 \mathrm{~cd}$ & 8.53 ef & $10.1 \mathrm{~g}$ & $21.8 \mathrm{abc}$ \\
\hline & $\mathrm{B} 2$ & $\mathrm{D} 2$ & $96.8 \mathrm{a}$ & 14.54 ef & $0.278 \mathrm{f}$ & 9.2 bcd & $11.27 \mathrm{~cd}$ & $23.8 \mathrm{ab}$ \\
\hline & $\mathrm{B} 2$ & D3 & $97.8 \mathrm{a}$ & $14.38 \mathrm{efg}$ & $0.251 \mathrm{fg}$ & $10.37 \mathrm{a}$ & $11.78 \mathrm{~b}$ & $24.4 \quad \mathrm{a}$ \\
\hline & B3 & D1 & $93.5 \mathrm{bc}$ & 14.53 ef & $0.32 \quad \mathrm{e}$ & $8.34 \mathrm{f}$ & $9.53 \mathrm{~h}$ & $21.4 \mathrm{abc}$ \\
\hline & B3 & $\mathrm{D} 2$ & 96 a.9 & 14.19 e-h & $0.258 \mathrm{fg}$ & 8.48 ef & $11.01 \mathrm{de}$ & 24 \\
\hline & B3 & D3 & $98.2 \mathrm{a}$ & $13.89 \mathrm{fgh}$ & $0.238 \mathrm{fg}$ & $10.44 \mathrm{a}$ & $11.84 \mathrm{ab}$ & $24.2 \mathrm{a}$ \\
\hline \multirow{9}{*}{ A2 } & B1 & D1 & $37.7 \mathrm{e}$ & $47 \quad c$ & $0.998 \quad b$ & $4.74 \mathrm{~h}$ & $6.66 \mathrm{j}$ & $14.8 \mathrm{bc}$ \\
\hline & B1 & $\mathrm{D} 2$ & $90.3 \mathrm{~d}$ & $15.74 \mathrm{~d}$ & $0.346 \mathrm{cde}$ & 8.71 def & $9.98 \mathrm{~g}$ & $17.2 \mathrm{abc}$ \\
\hline & B1 & D3 & $95 \mathrm{~b}$ & $14.02 \mathrm{e}-\mathrm{h}$ & $0.319 \mathrm{e}$ & $10.1 \mathrm{a}$ & $11.73 \mathrm{~b}$ & $22.2 \mathrm{abc}$ \\
\hline & $\mathrm{B} 2$ & D1 & $29.6 \mathrm{~g}$ & $53.44 \mathrm{a}$ & $1.066 \mathrm{a}$ & $3.83 \mathrm{i}$ & $6.97 \mathrm{j}$ & $13.4 \mathrm{c}$ \\
\hline & $\mathrm{B} 2$ & $\mathrm{D} 2$ & $89 \mathrm{~d}$ & $16.19 \mathrm{~d}$ & $\begin{array}{lll}0.38 & \mathrm{c} \\
\end{array}$ & 8.72 def & $10.24 \mathrm{~g}$ & $17.2 \mathrm{abc}$ \\
\hline & B2 & D3 & $94.7 \mathrm{~b}$ & $14.22 \mathrm{e}-\mathrm{h}$ & 0.359 cde & $9.48 \mathrm{~b}$ & $11.26 \mathrm{~cd}$ & $22.4 \mathrm{abc}$ \\
\hline & B3 & D1 & $34.9 \mathrm{f}$ & $49.11 \mathrm{~b}$ & $0.97 \quad b$ & $7.67 \mathrm{~g}$ & $8.69 \mathrm{i}$ & $16.4 \mathrm{abc}$ \\
\hline & B3 & $\mathrm{D} 2$ & $89 \mathrm{~d}$ & $15.93 \mathrm{~d}$ & $0.371 \mathrm{~cd}$ & $9.06 \mathrm{bcd}$ & $9.02 \mathrm{i}$ & $17.6 \mathrm{abc}$ \\
\hline & B3 & D3 & $94.3 \mathrm{~b}$ & $14.13 \mathrm{e}-\mathrm{h}$ & $0.341 \mathrm{cde}$ & $9.32 \mathrm{bc}$ & 10.71 ef & $21.6 \mathrm{abc}$ \\
\hline$S$ & & & ** & ** & ** & ** & ** & ** \\
\hline
\end{tabular}

${ }^{*},{ }^{* *}$, NS indicate $\mathrm{P}<0.05, \mathrm{P}<0.01$ and not significant, respectively. Means designated by the same letter within columns are not significantly different according to Duncan's multiple range test.

Data in Table 11 showed highly significant differences in all traits except acidity percentage due to the interaction among storage periods, environments and package materials. Results indicated that grains stored in jute bags and ash environment gave lower values in germination percentage, seedling vigor and higher values in electrical conductivity at the end of storage period. On the other hand grains stored in metal cans and dust or sand gave higher values in germination percentage, seedling vigor and lower values in electrical conductivity at the end of storage period. Similar results were recorded by Soares et al. (1993) and Tippes (1995).

Highly significant differences were found in radical and plumule length due to the interaction among cultivars, storage environments and package materials (Table 12). Data showed that grains stored in jute bags and ash environment gave lower values in radical length and plumule length with all cultivars especially Sakha 93, while grains stored in metal cans and dust or sand gave higher values for radical and plumule lengths with all cultivars. These results are in harmony with those reported by Soad (1997) and Govender et al. (2007).

The interaction among storage periods, cultivars, environments and package materials indicated highly significant differences in radical and plumule length (Tables 13and 14). Results indicated that grains stored in jute bags and ash environment gave the lowest radical and plumule lengths at the end of storage period with all cultivars especially sakha 93. On the other hand grains stored in metal cans and dust or sand gave the highest radical and plumule lengths with all cultivars at the end of storage period (18 month). Similar results were obtained by El-Borai et al. (1993), Soad (1997), El-Aidy et al. (2001) and El-Sayed et al. (2004b). 
Omar, A. M. et al.

Table 11: Germination, electrical conductivity and seedling vigor of wheat grains as affected by the interaction among storage periods, environments and package materials.

\begin{tabular}{|c|c|c|c|c|c|c|c|}
\hline Periods & Environments & $\begin{array}{l}\text { Package } \\
\text { materials }\end{array}$ & Germ. & E.C & $\begin{array}{c}\text { Radical } \\
\text { Length }(\mathrm{cm})\end{array}$ & $\begin{array}{c}\text { Plumule } \\
\text { Length }(\mathrm{cm})\end{array}$ & $\begin{array}{c}\text { Seedling } \\
\text { dry } \\
\text { weight(mg) }\end{array}$ \\
\hline \multirow{9}{*}{$A 1$} & C1 & D1 & 94 & $14.36 \mathrm{~d}$ & $9.26 \mathrm{~cd}$ & $10.31 d$ & 0.11 a-d \\
\hline & C1 & $\mathrm{D} 2$ & 97.5 a & $14.02 \mathrm{~d}$ & $9.19 \mathrm{~cd}$ & $11.62 \mathrm{ab}$ & $0.118 \mathrm{a}$ \\
\hline & C1 & D3 & 98.3 a & $13.87 \mathrm{~d}$ & $10.33 \mathrm{a}$ & $11.97 \mathrm{a}$ & $0.121 \mathrm{a}$ \\
\hline & C2 & D1 & $94 \quad c$ & $14.34 \mathrm{~d}$ & $8.26 \mathrm{fg}$ & $9.93 \mathrm{de}$ & $0.108 \mathrm{a}-\mathrm{d}$ \\
\hline & C2 & D2 & 97.5 a & $14.02 \mathrm{~d}$ & $9.53 \mathrm{c}$ & $11.28 \mathrm{bc}$ & $0.119 \mathrm{a}$ \\
\hline & $\mathrm{C} 2$ & D3 & 98.2 a & $13.86 \mathrm{~d}$ & $10.14 \mathrm{ab}$ & $11.92 \mathrm{a}$ & $0.119 \mathrm{a}$ \\
\hline & C3 & D1 & $91.8 \mathrm{~d}$ & $14.56 \mathrm{~d}$ & $8.28 \mathrm{fg}$ & $9.73 \mathrm{e}$ & $0.108 \mathrm{a}-\mathrm{d}$ \\
\hline & C3 & D2 & $96.5 \mathrm{ab}$ & $14.23 \mathrm{~d}$ & $8.02 \mathrm{~g}$ & $10.89 \mathrm{c}$ & $0.121 \mathrm{a}$ \\
\hline & C3 & D3 & $98.1 \quad \mathrm{a}$ & $13.87 \mathrm{~d}$ & 10.33 a & $11.98 \mathrm{a}$ & $0.12 \mathrm{a}$ \\
\hline \multirow{9}{*}{$A 2$} & C1 & D1 & $37.7 \mathrm{f}$ & $43.22 \mathrm{~b}$ & $5.97 \mathrm{~h}$ & $8.36 \mathrm{f}$ & 0.077 cde \\
\hline & C1 & $\mathrm{D} 2$ & 90.4 d & $15.76 \mathrm{c}$ & 8.66 ef & $9.99 \mathrm{de}$ & 0.09 a-e \\
\hline & C1 & D3 & 95 & $14.1 \quad \mathrm{~d}$ & $9.61 \mathrm{c}$ & $11.27 \mathrm{bc}$ & $0.108 \mathrm{a}-\mathrm{d}$ \\
\hline & C2 & D1 & $37.8 \mathrm{f}$ & $43.33 \mathrm{~b}$ & $5.57 \mathrm{~h}$ & $7.32 \mathrm{~g}$ & $0.076 \mathrm{de}$ \\
\hline & $\mathrm{C} 2$ & D2 & $90.3 \mathrm{~d}$ & $15.8 \mathrm{C}$ & $8.84 \mathrm{de}$ & $9.59 \mathrm{e}$ & $0.088 \mathrm{a}-\mathrm{e}$ \\
\hline & $\mathrm{C} 2$ & D3 & $94.3 \mathrm{c}$ & $14.14 \mathrm{~d}$ & $9.69 \mathrm{bc}$ & $11.13 \mathrm{c}$ & $0.111 \mathrm{abc}$ \\
\hline & C3 & D1 & $26.7 \mathrm{~g}$ & 63 & $4.71 \mathrm{i}$ & $6.63 \mathrm{~h}$ & $0.071 \mathrm{e}$ \\
\hline & C3 & $\mathrm{D} 2$ & $87.6 \mathrm{e}$ & $16.31 \mathrm{c}$ & 8.99 de & $9.67 \mathrm{e}$ & $0.081 \mathrm{~b}-\mathrm{e}$ \\
\hline & C3 & D3 & $94.7 \mathrm{bc}$ & $14.13 \mathrm{~d}$ & 9.6 & $11.3 \mathrm{bc}$ & $0.112 \mathrm{ab}$ \\
\hline & & & & & & & \\
\hline
\end{tabular}

${ }^{*}$, NS indicate $\mathrm{P}<0.05, \mathrm{P}<0.01$ and not significant, respectively. Means designated by the same lette within columns are not significantly different according to Duncan's multiple range test.

Table 12: Electrical conductivity, radical and plumule lengths of wheat grains as affected by the interaction among cultivars, storage environments and package materials.

\begin{tabular}{|c|c|c|c|c|}
\hline Cultivars & Environments & $\begin{array}{l}\text { Package } \\
\text { materials }\end{array}$ & $\begin{array}{c}\text { Radical } \\
\text { Length }(\mathbf{c m})\end{array}$ & $\begin{array}{c}\text { Plumule } \\
\text { Length }(\mathrm{cm})\end{array}$ \\
\hline \multirow{9}{*}{ B1 } & C1 & D1 & 7.4 & 9.4 \\
\hline & C1 & D2 & 8.5 efg & $10.92 \mathrm{dg}$ \\
\hline & C1 & D3 & $10.05 \mathrm{a}$ & $11.98 \mathrm{ab}$ \\
\hline & $\mathrm{C} 2$ & D1 & $5.9 \quad k$ & $7.783 \mathrm{p}$ \\
\hline & C2 & D2 & 9.38 & 10.73 e-h \\
\hline & $\mathrm{C} 2$ & D3 & $10.1 \quad \mathrm{a}$ & $12.02 \mathrm{a}$ \\
\hline & C3 & D1 & 7.18 hi & 8.3170 \\
\hline & C3 & D2 & $8.78 \quad c-f$ & $10.58 \mathrm{fgh}$ \\
\hline & C3 & D3 & $10 \quad a b$ & $11.97 \mathrm{ab}$ \\
\hline \multirow{9}{*}{ B2 } & C1 & D1 & $6.53 \mathrm{j}$ & $9.07 \mathrm{Imn}$ \\
\hline & $\mathrm{C} 1$ & D2 & $9.18 \mathrm{~cd}$ & 11.17 cde \\
\hline & C1 & D3 & $10.03 \mathrm{ab}$ & $11.55 \mathrm{abc}$ \\
\hline & C2 & D1 & 6.48 & $9.12 \quad \mathrm{Imn}$ \\
\hline & $\mathrm{C2}$ & D2 & 9.12 cde & $10.62 \mathrm{fgh}$ \\
\hline & C2 & D3 & $9.82 \mathrm{ab}$ & 11.45 bcd \\
\hline & C3 & D1 & $5.53 \mathrm{k}$ & $7.42 p$ \\
\hline & C3 & D2 & 8.58 d-g & $10.48 \mathrm{gh}$ \\
\hline & C3 & D3 & $9.92 \mathrm{ab}$ & $11.55 \mathrm{abc}$ \\
\hline \multirow{9}{*}{ B3 } & C1 & D1 & 8.9 & 9.53 \\
\hline & C1 & D2 & 9.08 cde & $10.33 \mathrm{hi}$ \\
\hline & $\mathrm{C} 1$ & D3 & $9.83 \mathrm{ab}$ & $11.32 \mathrm{~cd}$ \\
\hline & $\mathrm{C} 2$ & D1 & $8.35 \mathrm{fg}$ & $8.98 \mathrm{mn}$ \\
\hline & $\mathrm{C} 2$ & D2 & 9.07 cde & 9.95 \\
\hline & $\mathrm{C} 2$ & D3 & $9.83 \mathrm{ab}$ & $11.12 \mathrm{c}-\mathrm{f}$ \\
\hline & C3 & D1 & $6.77 \mathrm{ij}$ & 8.82 \\
\hline & C3 & D2 & $8.15 \mathrm{~g}$ & $9.77 \quad \mathrm{jk}$ \\
\hline & C3 & D3 & $9.98 \mathrm{ab}$ & $11.4 \mathrm{~cd}$ \\
\hline & Sig. & & & \\
\hline
\end{tabular}

${ }^{*},{ }^{\star *}$, NS indicate $\mathbf{P}<0.05, P<0.01$ and not significant, respectively. Means designated by the same letter within columns are not significantly different according to Duncan's multiple range test. 
Table 13: Radical length of wheat seedling as affected by the interaction among storage periods, cultivars, environments and package materials.

\begin{tabular}{|c|c|c|c|c|c|c|}
\hline \multirow[b]{2}{*}{ Periods } & \multirow[b]{2}{*}{ Cultivars } & \multirow{2}{*}{ Environments } & \multicolumn{4}{|c|}{ Package materials } \\
\hline & & & Jute & \multicolumn{2}{|c|}{$\begin{array}{l}\text { Jute coated } \\
\text { plastic }\end{array}$} & Metal Cans \\
\hline \multirow{9}{*}{6 months } & \multirow{3}{*}{ Gemmiza 7} & Dust & $9.27 \quad c-k$ & 8.4 & $\mathrm{k}-\mathrm{O}$ & $10.07 \quad \mathrm{a}-\mathrm{g}$ \\
\hline & & Sand & $8.27 \quad 1-0$ & 10.03 & $a-h$ & 10.1 \\
\hline & & Ash & 9.2 & 8.7 & $j-n$ & 9.83 \\
\hline & \multirow{3}{*}{ Sakha 93} & Dust & 9.5 & 10.07 & $a-j$ & $10.53 \mathrm{a}$ \\
\hline & & Sand & $8.37 \quad \mathrm{k}-\mathrm{o}$ & 9.4 & $c-j$ & 10.13 a-e \\
\hline & & Ash & 7.73 op & 8.13 & $\mathrm{~m}-\mathrm{o}$ & $10.43 \mathrm{ab}$ \\
\hline & \multirow{3}{*}{ Giza 168} & Dust & $i-m$ & 9.1 & $g-m$ & 10.4 \\
\hline & & Sand & $8.133 \mathrm{~m}-\mathrm{o}$ & 9.17 & e-I & 10.2 \\
\hline & & Ash & $n-p$ & 7.17 & $p$ & $10.73 \mathrm{a}$ \\
\hline \multirow{9}{*}{18 months } & \multirow{3}{*}{ Gemmiza 7} & Dust & $5.53 \mathrm{q}$ & 8.6 & j-o & 10.03 a-h \\
\hline & & Sand & $3.53 \mathrm{~s}$ & 8.733 & $j-n$ & $10.1 \quad a-f$ \\
\hline & & Ash & $5.17 \mathrm{qr}$ & 8.8 & j-n & 10.17 a-d \\
\hline & \multirow{3}{*}{ Sakha 93} & Dust & $3.57 \mathrm{~s}$ & 8.3 & $\mathrm{k}-\mathrm{o}$ & $9.53 \quad b-j$ \\
\hline & & Sand & 4.6 & 8.83 & $j-n$ & 9.5 \\
\hline & & Ash & $3.33 \mathrm{~s}$ & 9.03 & $\mathrm{i}-\mathrm{m}$ & 9.4 \\
\hline & \multirow{3}{*}{ Giza 168} & Dust & 8.8 & 9.07 & $\mathrm{~h}-\mathrm{m}$ & 9.27 \\
\hline & & Sand & $8.57 \mathrm{j}-\mathrm{o}$ & 8.97 & $\mathrm{i}-\mathrm{m}$ & 9.47 \\
\hline & & Ash & $5.63 \mathrm{q}$ & 9.13 & $f-I$ & 9.23 \\
\hline
\end{tabular}

Means designated by the same letter within columns are not significantly differen according to Duncan's multiple range test.

Table 14: Plumule length of wheat seedling as affected by the interaction among storage periods, cultivars, environments and package materials.

\begin{tabular}{|c|c|c|c|c|c|}
\hline \multirow{2}{*}{ Periods } & \multirow[b]{2}{*}{ Cultivars } & \multirow{2}{*}{ Environments } & \multicolumn{3}{|c|}{ Package materials } \\
\hline & & & Jute & $\begin{array}{l}\text { Jute coated } \\
\text { plastic }\end{array}$ & Metal Cans \\
\hline \multirow{9}{*}{6 months } & \multirow{3}{*}{ Gemmiza 7} & Dust & $10.63 \mathrm{~g}-\mathrm{k}$ & $11.77 \quad a-e$ & 12.33 \\
\hline & & Sand & $10.23 \quad \mathrm{i}-\mathrm{m}$ & 11.67 a-e & 12.23 \\
\hline & & Ash & $10.17 \mathrm{j}-\mathrm{n}$ & 11.1 & 12.17 \\
\hline & \multirow{3}{*}{ Sakha 93} & Dust & 10.43 h-m & 11.63 a-e & 11.7 \\
\hline & & Sand & $10.23 \quad \mathrm{i}-\mathrm{m}$ & $11.37 \quad c-g$ & 11.9 \\
\hline & & Ash & $9.63 \quad m-p$ & 10.8 & 11.73 \\
\hline & \multirow{3}{*}{ Giza 168} & Dust & 9.87 & 11.47 b-f & 11.87 \\
\hline & & Sand & 9.33 & 10.8 & 11.63 \\
\hline & & Ash & 9.4 & $10.77 \mathrm{f}-\mathrm{j}$ & 12.03 \\
\hline \multirow{9}{*}{18 months } & \multirow{3}{*}{ Gemmiza 7} & Dust & 8.17 & $10.07 \mathrm{j}-0$ & 11.63 a-e \\
\hline & & Sand & 5.33 & 9.8 & 11.8 \\
\hline & & Ash & 6.47 & $10.07 \mathrm{j}-0$ & 11.77 a-e \\
\hline & \multirow{3}{*}{ Sakha 93} & Dust & 7.7 & 10.7 & 11.4 \\
\hline & & Sand & st & $9.87 \quad k-p$ & $e-i$ \\
\hline & & Ash & 5.2 & $10.17 \mathrm{j}-\mathrm{n}$ & $11.37 \quad c-g$ \\
\hline & \multirow{3}{*}{ Giza 168} & Dust & 9.2 & 9.2 & $10.77 \mathrm{f}-\mathrm{j}$ \\
\hline & & Sand & 8.63 q-s & 9.1 & 10.6 \\
\hline & & Ash & $8.23 r-t$ & 8.77 & $10.77 \mathrm{f}-\mathrm{j}$ \\
\hline
\end{tabular}

Means designated by the same letter within columns are not significantly different according to Duncan's multiple range test. 


\section{CONCLUSION}

From the obtained results, it could be recommended to use metal cans to store grains for 18 months without using any chemicals. Grains stored with this method will remain germination percentage, viability and seedling vigor with a little decline.

\section{REFERENCES}

A. O. A. C (1999). Official Methods of Analysis, 8 th ed. Bass, L. N. (1971). Controlled atmosphere and seed storage. 16 th ISTACongress Washington D. C. June 7-12 TS 4-5.

Copeland, L. O., and M. B. Mc Donald (1995). Seed longevity and deterioration. In:Seed Science and Technol. pp. 181-220. (Chapman and Hill).

El-Aidy, N. A; S. A. El-Sayed and S.A. Salama. (2001). Effect of package kind on cotton seed quality and viability during storage. J. Agric. Res. Tanta Univ. ,27(4): 516-538.

El-Aidy, N. A. (1988). Optimizing storage conditions of rice grains. Candidate

in Agricultural Sciences. Odecca Technology Institute of food Technology, Odecca, USSR. (C.F.Computer Search).

El-Borai, M. A.; N. A. El-Aidy and M. El-Emery (1993).Effect of different storage periods on seed quality of three soybean cultivars. J. Agric. Sci., Mansoura Univ., 18(8):2206-2211.

El-Sayed, S. A. (1997). Effect of storage conditions on seed quality of wheat and faba bean. Ph. D. Thesis., Faculty of Agric., Kafr El-Sheikh, Tanta Univ., Egypt.Alex. J. Agric. Res., 50(2): 23-33.

El-Sayed, S. A.; M. E. A. Kineber and E. A. El-Kady. (2004a). Effect of storage environment and package material on storability, yield and its quality of flax seeds. J. Agric. Res. Tanta Univ. 30(3): 616-638.

El-Sayed, S. A.; M. E. A. Kineber and E. A. El-Kady. (2004b). Effect of package kind on cotton seed quality and viability during storage. $\mathrm{J}$. Agric. Res. Tanta Univ., 27(4): 326-340.

El-sayed, S. A. and M.N.Abd El-Aziz (2005). Relationship of viability parameters and quality of soybean under different storage condition. $J$. Agric. Res. Tanta Univ., 31 (4):526-538.

Gomez, K . A. and A . A. Gomez (1984). Statistical procedures for agricultural research. John Wiley and sons, Inc.,New york.

Govender,V.; T.A.S. Aveling and Q. Kritzinger(2007). The effect of traditional storage methods on germination and vigour of maize (Zea mays L.) from northern Kwazulu-Natal and southern Mozambique.South African Journal of Botany, 74: 190-196.

Harrington, J. F. and J. E. Douglas (1970). Seed storage and packaging. Applications for India. National Seeds Corporation Lth. In cooperation with the Rockefeller Foundation. New DIhi.(C.F.Computer Search).

Hong, M.; S. Song; Mc. Hong and S. Song (1994). Effects of packing method and storage temperature on the qualities of small packing milled rice. Bulletin of Taich. Agric. Impr. State. 43:5-17.

ISTA (2006). International rules for seed testing. Seed Sci. and Technol. Basserdorf, Switzerland.

Olakojo, S. A. and T.A. Akinlosotu (2004). Comparative study of storage ethods of maize grains in South Western Nigeria. African Journal of

Biotechnology 7: 362-365. 
Soares, N. D. F.; R. Cruz; S. T. Rezende; O. C. Soares and S. A. Crispim (1993). Effects of variety, packaging and period storage on brown rice quality. Arquivos de Biologia E Technologia, 36(3): 425-439.

Thamaga-Chitja, J.M.; S.L. Hendriks; G.F. Ortmann and M. Green (2004). Impact of maize storage on rural household food security in northen KwaZulu-Natal. Journal of Family Ecology and Consumer Science, 32: 8-15.(C.F.Computer Search).

Tipples, k. h. (1995). Quality and nutritional changes in stored grain. PP. 325351 in: Stored grain ecosystems, D. S. Jayas, J. White and W. E. Muir (eds.) Marcel Dekker, Inc., 270 Madison Avenue, New York. (C.F.Computer Search).

$$
\begin{aligned}
& \text { تاثير فترة التخزين والاصناف وبيئة التخزين ونوع العبوة المستخدمة على الإنبات }
\end{aligned}
$$

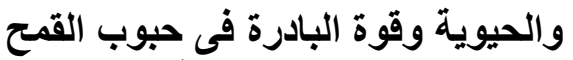

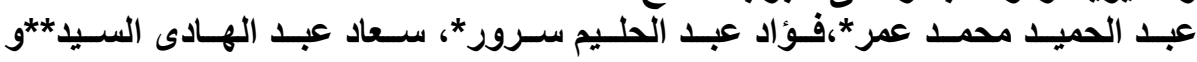

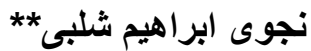

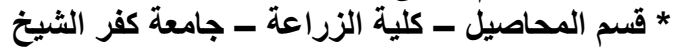

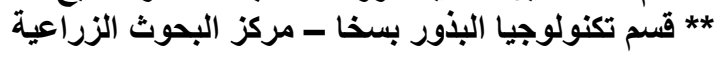

$$
\begin{aligned}
& \text { اجريت تجربة في محطة البحوث الزراعية بسخا لدراسة تا ثبر كل من فترات التخزين وانواع العبوات العبوات }
\end{aligned}
$$

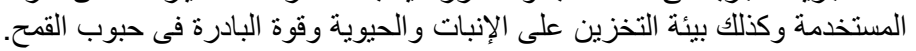

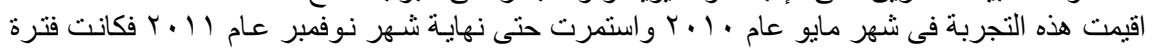

$$
\begin{aligned}
& \text { التخزين الكلية (1) الشهر). }
\end{aligned}
$$

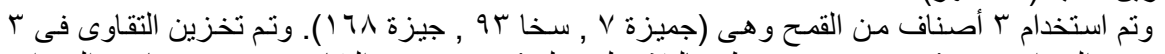

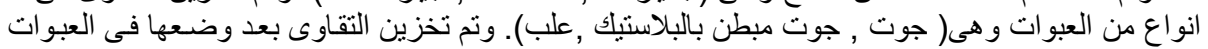

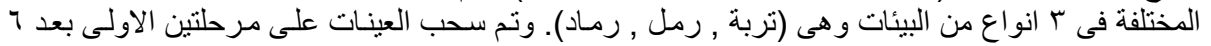

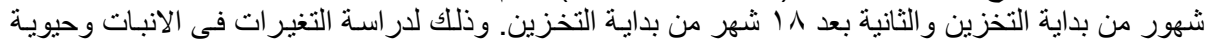

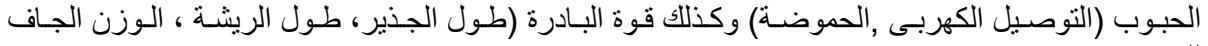

$$
\begin{aligned}
& \text { وقد تم دراسة جميع هذه الصفات قبل تخزين التقاوى وذلك للمقارنـة ومعرفة الاختلاف. واستخدم }
\end{aligned}
$$

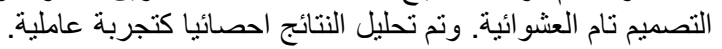

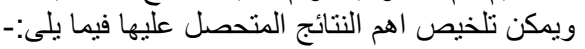

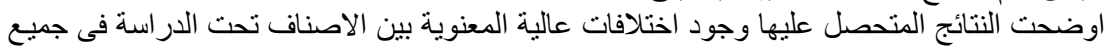

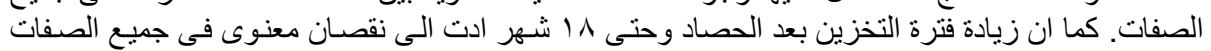

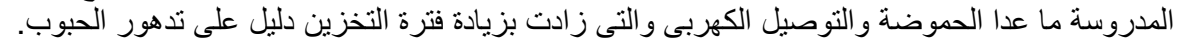

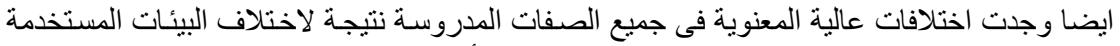

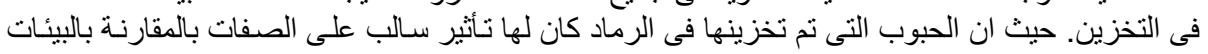

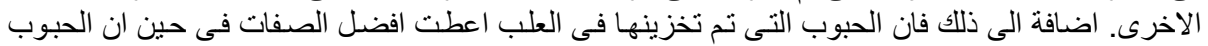

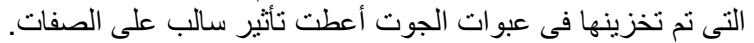

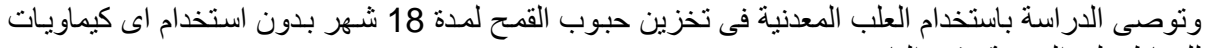

$$
\begin{aligned}
& \text { لل ل الحفاظ على الحيوية وقوة البادرة. }
\end{aligned}
$$

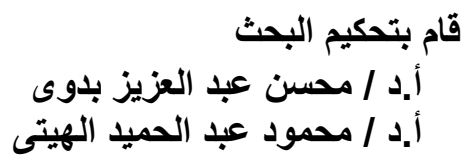

